Pacific Journal of Mathematic 


\title{
SOME PROPERTIES OF ALMOST RIMCOMPACT SPACES
}

\author{
BEVERLy DiAmond
}

\begin{abstract}
A 0 -space is a completely regular Hausdorff space possessing a compactification with zero-dimensional remainder. In a previous paper the class of almost rimcompact spaces was introduced and shown to be intermediate between the classes of rimcompact spaces and 0 -spaces. In this paper some properties of almost rimcompact spaces and of 0 -spaces are developed. If $X$ is a space whose non-locally compact part has compact boundary, then $X$ is a 0 -space if and only if $X$ is almost rimcompact. Neither perfect images or perfect preimages of rimcompact spaces need be 0 -spaces. However, if the perfect preimage of an almost rimcompact space is a 0 -space, then that perfect preimage is almost rimcompact. Subspaces and products are considered.
\end{abstract}

1. Introduction and known results. The characterization of those completely regular Hausdorff spaces possessing a compactification with zero-dimensional remainder has been considered by various researchers (see for example [7], [8] and [10]). Such a compactification will be called 0 -dimensional at infinity (denoted by O.I.); a 0-space is any space possessing a O.I. compactification. Recall that a space is rimcompact if it has a basis of open sets with compact boundaries ([7]). Each rimcompact space $X$ possesses a compactification which has a basis of open sets whose boundaries are contained in $X$ ([9], [10]), hence $X$ is a 0 -space; the converse is not true ([10]). In [2] we introduced a natural generalization of rimcompactness called almost rimcompactness and obtained the following characterization: a space $X$ is almost rimcompact if and only if $X$ possesses a compactification $K X$ in which each point of $K X \backslash X$ has a basis (in $K X$ ) of open sets whose boundaries are contained in $X$. (If $K X$ is such a compactification of $X$, we say that $K X \backslash X$ is relatively 0-dimensionally embedded in $K X$.) Hence each almost rimcompact space is a 0 -space; we showed in [2] that the converse is not true.

In this paper we discuss the properties of almost rimcompact spaces and of 0 -spaces. In $\S 2$ we show that if the non-locally compact part of $X$ has compact boundary, then $X$ is a 0 -space if and only if $X$ is almost rimcompact. Such a space need not be rimcompact. In $\$ 3$ we show that any closed subspace of a 0 -space (respectively, almost rimcompact space) 
is a 0 -space (respectively, almost rimcompact). This statement does not hold for open subspaces. In $\S 4$ we indicate that neither perfect images nor perfect preimages of rimcompact spaces need be 0 -spaces. However, if the perfect preimage of an almost rimcompact space is a 0 -space, then that perfect preimage is almost rimcompact.

In the remainder of this section, we present our notation and terminology and some known results. All spaces are assumed to be completely regular and Hausdorff. The notions used from set theory are standard. The symbol $\omega_{\alpha}$ is used to denote the $\alpha$ th cardinal. For any set $X$, $|X|$ denotes the cardinality of $X$. A map is a continuous surjection. A function $f: X \rightarrow Y$ is closed is whenever $F$ is a closed subset of $X$, then $f[F]$ is a closed subset of $Y$. A closed function $f: X \rightarrow Y$ is perfect if for each $y \in Y, f^{\leftarrow}(y)$ is compact.

The family $\mathscr{K}(X)$ of (equivalence classes of) compactifications of $X$ is partially ordered in the usual way: $J X \leq K X$ if there is a map $f: K X \rightarrow J X$ such that $f(x)=x$ for all $x \in X ; K X$ is equivalent to $J X$ if $f$ is a homeomorphism. For background information on compactifications the reader is referred to [1] or [4]. The maximum element of $K(X)$, the Stone-Čech compactification of $X$, is denoted by $\beta X$. In the sequel, if $K X \in \mathscr{K}(X)$, the natural map from $\beta X$ into $K X$ is denoted by $K f$.

The following is an easy consequence of 3.2.1 of [3].

1.1. Proposition (Taimanov's theorem). Let $K X$ and $K Y$ be compactifications of $X$ and $Y$ respectively, and $f$ be a map from $X$ into $Y$. There is $a$ map $f^{\prime}: K X \rightarrow K Y$ such that $\left.f^{\prime}\right|_{X}=f$ if and only if for $A, B \subset Y, \mathrm{Cl}_{K Y} A \cap$ $\mathrm{Cl}_{K Y} B=\varnothing$ implies $\mathrm{Cl}_{K X} f^{\leftarrow}[A] \cap \mathrm{Cl}_{K X} f^{\leftarrow}[B]=\varnothing$.

The next result follows from 1.5 of [6].

1.2. Proposition. Let $X, Y, K X, K Y$ and $f$ be as in 1.1. If $f$ is perfect, and if $f^{\prime}$ exists, then $f^{\prime}(K X \backslash X)=K Y \backslash Y$.

We often call $K X \backslash X$ the remainder of $K X$. For any space $X$, the residue of $X$ (denoted by $R(X)$ ) is the set of points at which $X$ is not locally compact. If $K X$ is any compactification of $X$, then $\mathrm{Cl}_{K X}(K X \backslash X)$ $=R(X) \cup(K X \backslash X)$.

The first of the following results combines Theorems 1 and 4 of [5]; the second is 6.7 of [4]. 
1.3. Proposition. Let $\left\{X_{\alpha}: \alpha \in A\right\}$ be a set of pseudocompact spaces. Then:

(i) If $\Pi\left\{X_{\alpha}: \alpha \in A\right\}$ is pseudocompact, then $\beta\left[\Pi\left\{X_{\alpha}: \alpha \in A\right\}\right]=$ $\Pi\left\{\beta X_{\alpha}: \alpha \in A\right\}$.

(ii) If $X_{\alpha}$ is locally compact for all but one $\alpha \in A$, then $\prod\left\{X_{\alpha}: \alpha \in A\right\}$ is pseudocompact.

1.4. Proposition. If $X$ is any space, and $X \subset T \subset \beta X$, then $\beta T=\beta X$.

If $U$ is an open subset of $X$, and $\delta X \in \mathscr{K}(X)$, then $E x_{\delta X} U$ is defined to be $\delta X \backslash \mathrm{Cl}_{\delta X}(X \backslash U)$. The set $E x_{\delta X} U$ is often called the extension of $U$ in $\delta X$. It is an easy exercise to verify (i), (ii), and (iii) of the following proposition. Statement (iv) is implicit in the proof of Lemma 2 of [10].

\subsection{Proposition. Let $\delta X \in \mathscr{K}(X)$.}

(i) If $W$ is open in $\delta X$, then $W \subset E x_{\delta X}(W \cap X)$.

(ii) If $U$ and $V$ are open in $X$, then $E x_{\delta X}(U \cap V)=\left(E x_{\delta X} U\right) \cap$ $\left(E x_{\delta X} V\right)$.

(iii) If $U$ is open in $X$, then $\left(E x_{\delta X} U\right) \cap X=U$, hence $\mathrm{Cl}_{\delta X} U=$ $\mathrm{Cl}_{\delta X} E x_{\delta X} U$.

(iv) If $U$ and $V$ are open in $X$, then

$$
E x_{\delta X}(U \cup V) \backslash\left(E x_{\delta X} U \cup E x_{\delta X} V\right) \subset \mathrm{Cl}_{\delta X} U \cap \mathrm{Cl}_{\delta X} V .
$$

If $U$ is any open subset of $X$, then it follows from 1.5(i) that $E x_{\delta X} U$ is the largest open subset of $\delta X$ whose intersection with $X$ is the set $U$. The collection $\left\{E x_{\delta X} U: U\right.$ is an open subset of $\left.X\right\}$ of open sets of $\delta X$ is easily seen to be a basis for the topology of $\delta X$.

If $B \subset X$, the boundary of $B$ in $X$, denoted by $\mathrm{bd}_{X} B$, is defined to be the set $\mathrm{Cl}_{X} B \cap \mathrm{Cl}_{X}(X \backslash B)$. A compactification $\delta X$ of $X$ is a perfect compactification of $X$ if for each open subset of $U$ of $X, \mathrm{Cl}_{\delta X}\left(\mathrm{bd}_{X} U\right)=$ $\operatorname{bd}_{\delta X}\left(E x_{\delta X} U\right)$. According to the corollary to Lemma 1 of [10], $\beta X$ is a perfect compactification of $X$.

The equivalence of (i), (ii), and (iii) of the following proposition appear in Theorems 1 and 2 of [10].

1.6. Proposition. Let $\delta X \in \mathscr{K}(X)$. The following are equivalent.

(i) $\delta X$ is a perfect compactification of $X$.

(ii) If $U$ and $V$ are disjoint open sets of $X$, then $E_{\delta X}(U \cup V)=$ $E x_{\delta X} U \cup E x_{\delta X} V$.

(iii) For each $p \in \delta X,(\delta f)^{\leftarrow}(p)$ is a connected subset of $\beta X$. 
The connected component $C_{x}$ of $x \in X$ is the union of all connected subspaces of $X$ containing $x$. A space $X$ is totally disconnected if $C_{x}=\{x\}$ for each $x \in X$. The quasi-component of $x \in X$ is the intersection of all closed-and-open (denoted clopen) subsets of $X$ containing $x$. A space $X$ is zero-dimensional (denoted 0-dimensional) if $X$ has a basis of clopen sets. A space $X$ is strongly 0-dimensional if any two disjoint zerosets of $X$ are contained in disjoint clopen subsets of $X$.

For a detailed discussion of the disconnectedness of remainders of compactifictions see [2]. Any 0 -space $X$ has a maximum O.I. compactification (which we denote by $F_{0} X$ ) which is also a minimum perfect compactification of $X$. For each $p \in F_{0} X \backslash X$, the set $\left(F_{0} f\right)^{\leftarrow}(p)$ is the connected compact quasi-component (in $\beta X \backslash X$ ) of each element of $\left(F_{0} f\right) \leftarrow(p)$.

The maximum O.I. compactification of a rimcompact space $X$ is called the Freudenthal compactification of $X$, and is denoted by $F X$. If $X$ is 0 -dimensional then $F X=\beta_{0} X$, where $\beta_{0} X$ denotes the maximum 0-dimensional compactification of $X$.

Following the terminology of [9] and [10], we say that an open set $U$ of $X$ is $\pi$-open in $X$ if $b_{X} U$ is compact. The intersection and union of finitely many $\pi$-open sets are $\pi$-open, as is the complement of the closure of a $\pi$-open set.

1.7. Definitions. (i) If $F_{1}, F_{2} \subset X$, then $F_{1}$ and $F_{2}$ are $\pi$-separated in $X$ if there is a $\pi$-open set $U$ of $X$ such that $F_{1} \subset U$, and $\mathrm{Cl}_{X} U \cap F_{2}=\varnothing$. We shall often write " $\{x\}$ and $F$ are $\pi$-separated" as " $x$ and $F$ are $\pi$-separated". We say that $F_{1}$ is $\pi$-contained in $X \backslash F_{2}$ if $F_{1}$ and $F_{2}$ are $\pi$-separated.

(ii) If $F$ is closed in $X, U$ is open in $X$, and $F \subset U$, then $F$ is nearly $\pi$-contained in $U$ if there is a compact subset $K$ of $F$ so that whenever $F^{\prime}$ is a closed subset of $F$, and $F^{\prime} \cap K=\varnothing, F^{\prime}$ is $\pi$-contained in $U$.

(iii) A space $X$ is nearly rimcompact if whenever $U$ is open in $X$, and $x \in U$, there is an open set $W$ of $X$ such that $x \in W$ and $\mathrm{Cl}_{X} W$ is nearly $\pi$-contained in $U$.

(iv) A space $X$ is quasi-rimcompact if for any $x \in X$, there is a compact set $K_{x}$ of $X$, so that whenever $F$ is a closed subset of $X$ and $F \cap K_{x}=\varnothing$, then $x$ and $F$ are $\pi$-separated.

(v) A space $X$ is almost rimcompact if $X$ is nearly rimcompact and quasi-rimcompact.

The following is 2.18 of [2]. 
1.8. THEOREM. For any space $X$, the following are equivalent.

(i) $X$ is almost rimcompact.

(ii) $X$ is a 0 -space, and $F_{0} X$ has relatively 0 -dimensionally embedded remainder.

(iii) $X$ has a compactification with relatively 0 -dimensionally embedded remainder.

(iv) $X$ is quasi-rimcompact, and has a compactification with totally disconnected remainder.

The following is justified in 3.5 of [2].

1.9. EXAMPLe. Let $Y$ be any 0 -dimensional non-strongly 0 -dimensional space, and let $K Y$ be any perfect compactification of $Y$. If $X=$ $\left(K Y \times\left(\omega_{1}+1\right)\right) \backslash\left(Y \times\left\{\omega_{1}\right\}\right)$, then $X$ is almost rimcompact. $X$ is rimcompact if and only if $K Y=\beta_{0} Y$.

2. 0 -spaces whose residues have compact boundary. We begin by listing some straightforward results concerning $\pi$-open subsets of $X$ and related open subsets of compactifications of $X$.

2.1. Definition. Let $K X \in \mathscr{K}(X)$, and let $W$ be open in $K X$. If $\mathrm{bd}_{K X} W \subset X, W$ is said to be a small boundary (denoted by sb) subset of $K X$.

2.2. LemMa. Let $K X \in \mathscr{K}(X)$.

(i) The intersection (union) of finitely many sb open subsets of $K X$ is an sb open subset of $K X$.

If $W$ is an sb open subset of $K X$, then

(ii) $W \cap X$ is $\pi$-open in $X$.

(iii) $W=E x_{K X}(W \cap X)$.

(iv) $K X \backslash \mathrm{Cl}_{K X} W$ is sb in $K X$.

(v) If $U$ is $\pi$-open in $X$, and $K X$ is a perfect compactification of $X$, then $\mathrm{Cl}_{K X} U \cap(K X \backslash X)=E x_{K X} U \cap(K X \backslash X)$; that is, $E x_{K X} U$ is an sb open subset of $K X$.

The straightforward proof of 2.2 is left to the reader.

We consider separately the cases where $X$ is nowhere locally compact, and where $X$ has compact residue.

2.3. LemMA. Suppose that $X$ is nowhere locally compact, and that $K X$ is a O.I. compactification of $X$. Then $K X \backslash X$ is relatively 0-dimensionally embedded in $K X$. 
Proof. Suppose that $p \in K X \backslash X$, and that $p \in W$, where $W$ is an open subset of $K X$. Since $K X \backslash X$ is 0 -dimensional, there is a clopen subset $V$ of $K X \backslash X$ such that $p \in V \subset W \cap(K X \backslash X)$, and $\mathrm{Cl}_{K X} V \subset W$. Let $U$ be any open subset of $K X$ such that $U \cap(K X \backslash X)=V$. Since $K X \backslash X$ is dense in $K X, \mathrm{Cl}_{K X} U=\mathrm{Cl}_{K X}(U \cap(K X \backslash X))=\mathrm{Cl}_{K X} V$. Then

$$
\left(\mathrm{Cl}_{K X} U\right) \cap(K X \backslash X)=\mathrm{Cl}_{K X} V \cap(K X \backslash X)=\mathrm{Cl}_{K X \backslash X} V=V .
$$

It follows that $\mathrm{bd}_{K X} U=\mathrm{Cl}_{K X} U \backslash U \subset X$, hence $U$ is an sb open subset of $K X$. Since $\mathrm{Cl}_{K X} U \subset W, p$ has a basis in $K X$ of sb open sets of $K X$. Thus $K X \backslash X$ is relatively 0 -dimensionally embedded in $K X$.

We make the following easily proved result explicit.

2.4. Lemma. Suppose that $S, T$ are closed subsets of $X$, and that $S \cap(T \cup R(X))=\varnothing$. If $S$ is compact, then there is an open set $U$ of $X$ such that $\mathrm{Cl}_{X} U$ is compact, $S \subset U$, and $T \cap \mathrm{Cl}_{X} U=\varnothing$.

2.5. Lemma. Let $X$ be a space, and let $K X \in \mathscr{K}(X)$. Suppose that $T$ is a closed subset of $K X$, that $W$ is a compact clopen subset of $\mathrm{Cl}_{K X}(K X \backslash X)$ and that $T \cap W=\varnothing$. Then there is an sb open set $U$ of $K X$ such that $\mathrm{bd}_{K X} U \subset X \backslash R(X), W=U \cap \mathrm{Cl}_{K X}(K X \backslash X)$, and $T \cap \mathrm{Cl}_{K X} U=\varnothing$.

Proof. If $W$ is a compact clopen subset of $\mathrm{Cl}_{K X}(K X \backslash X)$, then $W^{\prime}=\mathrm{Cl}_{K X}(K X \backslash X) \backslash W$ is a compact clopen subset of $\mathrm{Cl}_{K X}(K X \backslash X)$. There are disjoint open sets $U_{1}, U_{1}^{\prime}$ of $K X$ such that $W \subseteq U_{1}, W^{\prime} \subseteq U_{1}^{\prime}$ and $\mathrm{Cl}_{K X} U_{1} \cap \mathrm{Cl}_{K X} U_{1}^{\prime}=\varnothing$. Then $\mathrm{bd}_{K X} U_{1} \subseteq X \backslash R(X)$, hence $U_{1}$ is an sb open subset of $K X$. Also, $U_{1} \cap \mathrm{Cl}_{K X}(K X \backslash X)=W$. Since $T \cap W=$ $\varnothing$, it follows that $T \cap \mathrm{Cl}_{K X}(K X \backslash X) \cap \mathrm{Cl}_{K X}\left(U_{1} \cap X\right)=\varnothing$, hence $T \cap$ $\mathrm{Cl}_{K X}\left(U_{1} \cap X\right)$ is a compact set contained in $X \backslash R(X)$. According to 2.4, there is an open set $V$ of $X$ such that $\mathrm{Cl}_{X} V$ is a compact subset of $X \backslash R(X)$, and $T \cap \mathrm{Cl}_{K X}\left(U_{1} \cap X\right) \subset V$. Let $U_{2}=K X \backslash \mathrm{Cl}_{X} V$. Then $U_{2}$ is an sb open set of $K X$ by 2.2 (iv), and $W \subset U_{2}$. If $U=U_{1} \cap U_{2}$, then $U$ is an sb open set of $K X$ by $2.2(\mathrm{i})$, and $W=U \cap \mathrm{Cl}_{K X}(K X \backslash X)$. Also $\mathrm{bd}_{K X} U \subset \mathrm{bd}_{K X} U_{1} \cup \mathrm{bd}_{K X} U_{2} \subset X \backslash R(X)$. Since $T \cap \mathrm{Cl}_{K X} U \subset T \cap$ $\mathrm{Cl}_{K X} U_{1} \cap \mathrm{Cl}_{K X} U_{2}=\varnothing$, the statement is proved.

Let $X$ be a space. In the sequel, $L(X)$ denotes the locally compact part of $X$; that is $L(X)=X \backslash R(X)$. Note that if $K X \in \mathscr{K}(X)$, then $L(X)=K X \backslash \mathrm{Cl}_{K X}(K X \backslash X)$, and that

$$
L(K X \backslash X)=(K X \backslash X) \backslash R(K X \backslash X)=K X \backslash\left[X \cup \mathrm{cl}_{K X} R(X)\right] .
$$


The following is easy to prove.

2.6. LEMMA. If $X$ is a space, $K X \in \mathscr{K}(X)$, and $W$ is a compact clopen subset of either $L(K X \backslash X)$ or $K X \backslash X$, then $W$ is a (compact) clopen subset of $\mathrm{Cl}_{K X}(K X \backslash X)$.

2.7. Lemma. Suppose that $X$ is a space in which $R(X)$ is compact. If $K X$ is a O.I. compactification of $X$, then $K X \backslash X$ is relatively 0-dimensionally embedded in $K X$.

Proof. Suppose that $T$ is closed in $K X$, and that $p \in(K X \backslash X) \backslash T$. As $R(X)$ is compact, there is an open set $U$ of $K X$ such that $p \in U$, while $[T \cup R(X)] \cap \mathrm{Cl}_{K X} U=\varnothing$. Since $U \cap(K X \backslash X)$ is open in $K X \backslash X$, and $K X \backslash X$ is locally compact and 0 -dimensional, there is a compact clopen set $W$ of $K X \backslash X$ such that $p \in W \subset U$. Then $W \cap T=\varnothing$, so by 2.5 and 2.6 there is an sb open set $V$ of $K X$ such that $V \cap \mathrm{Cl}_{K X}(K X \backslash X)=W$ and $T \cap \mathrm{Cl}_{K X} V=\varnothing$. Then $p \in V$, and $V \cap T=\varnothing$. Thus each point of $K X \backslash X$ has a basis in $K X$ of open sets whose boundaries lie in $X$. That is, $K X \backslash X$ is relatively 0-dimensionally embedded in $K X$.

2.8. TheOREM. If $X$ is a space in which $\mathrm{bd}_{X} R(X)$ is compact, then the following are equivalent.

(i) $X$ is a 0-space.

(ii) $X$ is almost rimcompact.

(iii) $X$ is a 0 -space, and $F_{0} X \backslash X$ is relatively 0 -dimensionally embedded in $F_{0} X$.

(iv) If $K X$ is any O.I. compactification of $X$ in which $\mathrm{Cl}_{K X}\left(\operatorname{int}_{X} R(X)\right)$ $\cap \mathrm{Cl}_{K X}(X \backslash R(X)) \subset X$, then $K X \backslash X$ is relatively 0-dimensionally embedded in $K X$.

Proof. It follows from 1.8 that (iii) implies (ii) and (ii) implies (i).

(i) implies (iv). Suppose that $K X$ is a O.I. compactification of $X$ in which $\mathrm{Cl}_{K X}\left(\right.$ int $\left._{X} R(X)\right) \cap \mathrm{Cl}_{K X}(X \backslash R(X)) \subset X$. We claim that

$$
K X \backslash X \subset E x_{K X}\left(\operatorname{int}_{X} R(X)\right) \cup E x_{K X}(X \backslash R(X)) \text {. }
$$

As $X \backslash\left[\operatorname{int}_{X} R(X) \cup(X \backslash R(X))\right]=\mathrm{bd}_{X} R(X)$, which is a compact subset of $X$,

$$
K X \backslash X \subset E x_{K X}\left[\operatorname{int}_{X} R(X) \cup(X \backslash R(X))\right] .
$$

If $U$ and $V$ are open subsets of $X$, and

$$
p \in E x_{K X}(U \cup V) \backslash\left(E x_{K X} U \cup E x_{K X} V\right),
$$


then by 1.5 (iv), $p \in \mathrm{Cl}_{K X} U \cap \mathrm{Cl}_{K X} V$. As

$$
\mathrm{Cl}_{K X}\left(\operatorname{int}_{X} R(X)\right) \cap \mathrm{Cl}_{K X}(X \backslash R(X)) \subset X,
$$

it follows that $K X \backslash X \subset E x_{K X}\left(\right.$ int $\left._{X} R(X)\right) \cup E x_{K X}(X \backslash R(X))$, and the claim is proved.

Note that $\mathrm{Cl}_{X}$ int ${ }_{X} R(X)$ is a nowhere locally compact space. For if $V$ is any non-empty open subset of $\mathrm{Cl}_{X}$ int $_{X} R(X)$, there is an open set $U$ of $X$ such that

$$
U \cap \mathrm{Cl}_{X} \operatorname{int}_{X} R(X)=V .
$$

Then $U \cap$ int $_{X} R(X)$ is a non-empty open subset of $X$. Since int ${ }_{X} R(X)$ is nowhere locally compact, $\mathrm{Cl}_{X}\left(U \cap\right.$ int $\left._{X} R(X)\right)$ is not compact. Then $\mathrm{Cl}_{X} V$, which is the closure in $\mathrm{Cl}_{X}$ int ${ }_{X} R(X)$ of $V$, is not compact. Thus no point of $\mathrm{Cl}_{X}$ int $_{X} R(X)$ has a basis (in $\mathrm{Cl}_{X}$ int ${ }_{X} R(X)$ ) of compact closed neighbourhoods, and $\mathrm{Cl}_{X}$ int ${ }_{X} R(X)$ is nowhere locally compact.

As $\mathrm{Cl}_{K X}$ int ${ }_{X} R(X)$ is a O.I. compactification of $\mathrm{Cl}_{X}$ int $_{X} R(X)$, it follows from 2.3 that $\mathrm{Cl}_{K X}$ int $_{X} R(X) \backslash \mathrm{Cl}_{X}$ int ${ }_{X} R(X)$, which by our claim is just [Ex $x_{K X}$ int $\left._{X} R(X)\right] \cap[K X \backslash X]$, is relatively 0-dimensionally embedded in $\mathrm{Cl}_{K X}$ int ${ }_{X} R(X)$. Let $p \in\left[E x_{K X}\right.$ int $\left._{X} R(X)\right] \cap[K X \backslash X]$. We show that $p$ has a basis in $K X$ of open sets whose boundaries lie in $X$. Suppose that $p \in K X \backslash T$, where $T$ is a closed subset of $K X$. Since $p \notin \mathrm{Cl}_{K X}(X \backslash R(X))$, there is an open subset $U_{1}$ of $K X$ such that $p \in U_{1}$ and $\mathrm{Cl}_{K X} U_{1} \cap\left[\mathrm{Cl}_{K X}(X \backslash R(X)) \cup T\right]=\varnothing$. Then $U_{1}$ is open in $E x_{K X}$ int $_{X} R(X)$, and hence in $\mathrm{Cl}_{K X}$ int ${ }_{X} R(X)$. It follows that there is an sb (with respect to $\mathrm{Cl}_{K X}$ int ${ }_{X} R(X)$ ) open set $U_{2}$ of $\mathrm{Cl}_{K X}$ int ${ }_{X} R(X)$ such that $p \in U_{2} \subset U_{1}$. As $U_{1} \subset E x_{K X}$ int $_{X} R(X)$, it follows that $U_{2}$ is open in $K X$. Since $\mathrm{Cl}_{K X} U_{2} \cap \mathrm{Cl}_{K X}(X \backslash R(X))=\varnothing, U_{2}$ is an sb open subset of $K X$ which contains $p$ and has empty intersection with $T$.

The subset $\mathrm{Cl}_{X}(X \backslash R(X))$ of $X$ is a space with compact residue, so by $2.7, \mathrm{Cl}_{K X}(X \backslash R(X))$ is a O.I. compactification of $X$ with a relatively 0 -dimensionally embedded remainder. If

$$
p \in \mathrm{Cl}_{K X}(X \backslash R(X)) \backslash \mathrm{Cl}_{X}(X \backslash R(X))
$$

(which by our earlier claim equals $\left.E x_{K X}(X \backslash R(X)) \cap(K X \backslash X)\right)$, then $p \notin \mathrm{Cl}_{K X} R(X)$. It follows from an argument similar to that in the preceding paragraph that $p$ has a basis in $K X$ of sb open sets of $K X$. Thus each point of $K X \backslash X$ has a basis of sb open sets of $K X$, hence $K X \backslash X$ is relatively 0 -dimensionally embedded in $K X$. 
(iv) implies (iii). Since $F_{0} X$ is a perfect compactification of $X$, and bd $_{X} R(X)$ is compact, by $2.2(\mathrm{v})$ and 1.5 (ii),

$$
\begin{aligned}
& \mathrm{Cl}_{F_{0} X}\left(\text { int }_{X} R(X)\right) \cap \mathrm{Cl}_{F_{0} X}(X \backslash R(X)) \cap\left(F_{0} X \backslash X\right) \\
& \quad=E x_{F_{0} X} \text { int }_{X} R(X) \cap E x_{F_{0} X}(X \backslash R(X)) \cap\left(F_{0} X \backslash X\right)=\varnothing .
\end{aligned}
$$

Thus $F_{0} X$ satisfies the condition imposed on $K X$ in (iv) and hence $F_{0} X \backslash X$ is relatively 0 -dimensionally embedded in $F_{0} X$.

The hypotheses of 2.8 do not imply that $X$ is rimcompact. If in $1.9, Y$ is chosen to be a locally compact 0-dimensional space which is not strongly 0 -dimensional, and $\beta Y$ is chosen as the perfect compactification of $Y$, then $X=\left(\beta Y \times\left(\omega_{1}+1\right)\right) \backslash\left(Y \times\left\{\omega_{1}\right\}\right)$ is an almost rimcompact non-rimcompact space in which $R(X)$ is compact.

3. Subsets, supersets and products. We outline a construction that we will use to produce many of our examples.

A collection of infinite subsets of $\mathscr{N}$ is called almost disjoint if the intersection of the two distinct members is finite. Zorn's lemma implies that there exists a maximal collection of almost disjoint infinite subsets of $\mathscr{N}$. In the following $\mathscr{R}$ will denote a maximal such collection. The following topology on $\mathscr{N} \cup \mathscr{R}$ is credited to Isbell in [4]. Each point of $\mathscr{N}$ is isolated, and $\lambda \in \mathscr{R}$ has as an open base $\{\{\lambda\} \cup(\lambda \backslash F): F$ is a finite subset of $\mathscr{N}$. It is noted in 5I of [4] that such spaces $\mathscr{N} \cup \mathscr{R}$ are first countable, locally compact, 0-dimensional and pseudocompact. The following is 2.1 of [12].

3.1. Proposition. Any compact metric space without isolated points is homeomorphic to the remainder $\beta(\mathscr{N} \cup \mathscr{R}) \backslash \mathscr{N} \cup \mathscr{R}$ for a suitably chosen maximal almost disjoint collection $\mathscr{R}$.

As indicated in [12], 3.1 holds for any first-countable, separable, compact $T_{2}$ space. We do not make use of this more general statement.

In the sequel, when we choose a maximal almost disjoint collection $\mathscr{R}$ such that $\beta(\mathscr{N} \cup \mathscr{R}) \backslash \mathscr{N} \cup \mathscr{R}$ is homeomorphic to a compact metric space $X$ having no isolated points, we identify points of

$$
\beta(\mathscr{N} \cup \mathscr{R}) \backslash \mathscr{N} \cup \mathscr{R}
$$

with points of $X$ in the obvious manner, and consider $\beta(\mathscr{N} \cup \mathscr{R}) \backslash \mathscr{N} \cup \mathscr{R}$ to be the space $X$. 
The next example shows that, as might be expected, it is not true that if a space $X$ is rimcompact, and $X \subset T \subset \beta X$, then $T$ is necessarily a 0 -space.

3.2. EXAmple. Choose $\mathscr{R}$ so that $\beta(\mathscr{N} \cup \mathscr{R}) \backslash \mathscr{N} \cup \mathscr{R}=I$, where $I$ denotes the unit interval. Let $X=\mathscr{N} \cup \mathscr{R}$, and $T=\mathscr{N} \cup \mathscr{R} \cup\{1\}$. Then $X$ is rimcompact. However, the single connected component of $\beta T \backslash T=$ $\beta X \backslash T$ is $[0,1)$, which is not compact. Thus $T$ is not a 0 -space.

It is clear that if $X$ is a 0 -space, and $X \subset T \subset F_{0} X$, then $T$ is a 0 -space. Recall that if $X \subset Y \subset \beta X$, then $\beta Y=\beta X$. The following indicates that the expected relationship between $F_{0} X$ and $F_{0} T$ holds.

3.3. THEOREM. If $X$ is a 0 -space, and $X \subset T \subset F_{0} X$, then $T$ is a 0 -space and $F_{0} X=F_{0} T$. If $X$ is almost rimcompact (respectively, rimcompact) then $T$ is almost rimcompact (respectively, rimcompact).

Proof. Clearly $F_{0} X$ is a O.I. compactification of $T$. Suppose that $K T$ is a O.I. compactification of $T$ such that $K T \geq F_{0} X$. Then $K T$ is a compactification $\delta X$ of $X$. Recall that $\delta f: \beta X \rightarrow \delta X$ denotes the natural map. Define $g: \delta X \rightarrow F_{0} X$ to be the natural map. Then $g \circ(\delta f)=F_{0} f$. Suppose that $p \in F_{0} X \backslash T$. Since $F_{0} X$ is a perfect compactification of $X$, by 1.6, $\left(F_{0} f\right)^{\leftarrow}(p)=(g \circ \delta f)^{\leftarrow}(p)$ is a connected subset of $\beta X$. Then $(\delta f)\left[\left(F_{0} f\right) \leftarrow(p)\right]=g^{\leftarrow}(p)$ is a connected subset of $K T$ contained in $K T \backslash T$. Since $K T \backslash T$ is 0 -dimensional, $\left|g^{\leftarrow}(p)\right|=1$. It follows that $K T=F_{0} X$, and hence $F_{0} X=F_{0} T$.

If each point of $F_{0} X \backslash X$ has a basis of open sets of $F_{0} X$ whose boundaries are contained in $X$, then each point of $F_{0} X \backslash T$ has a basis of open sets of $F_{0} X=F_{0} T$ whose boundaries are contained in $T$. Thus if $X$ is almost rimcompact, $T$ is almost rimcompact. A similar statement holds if $X$ is rimcompact.

It is tempting to attempt to shorten the proof of the preceding theorem by immediately claiming that $K T$ as chosen is a O.I. compactification of $X$. However, since the union of two 0-dimensional spaces need not be 0 -dimensional, it is not immediately clear that $K T \backslash X$ is 0 -dimensional, and further argument of the sort provided in the proof is necessary.

We note in passing the following special case for 3.3. If $X$ is a 0 -space, and $X \cup \mathrm{Cl}_{F_{0} X} R(X) \subset T \subset F_{0} X$, then since $X \cup \mathrm{Cl}_{F_{0} X} R(X)$ is almost rimcompact by $2.7, T$ is almost rimcompact. 
We now consider subspaces of 0 -spaces. It is an easy exercise to prove that an open or a closed subspace of a rimcompact space is rimcompact. This contrasts with the fact that while a closed subspace of an almost rimcompact space is almost rimcompact, an open subspace of an almost rimcompact space need not even be a 0 -space.

3.4. THEOREM. If $F$ is a closed subset of a 0-space (respectively, an almost rimcompact space) $X$, then $F$ is a 0 -space (respectively, almost rimcompact).

Proof. If $F$ is closed in a 0 -space $X$, and $K X$ is any O.I. compactification of $X$, then $\mathrm{Cl}_{K X} F$ is a O.I. compactification of $F$. Thus $F$ is a 0 -space.

Suppose that $K X \backslash X$ is relatively 0 -dimensionally embedded in $K X$. We show that $\mathrm{Cl}_{K X} F \backslash F$ is relatively 0 -dimensionally embedded in $\mathrm{Cl}_{K X} F$. Suppose that $T$ is a closed subset of $\mathrm{Cl}_{K X} F$ and $p \in\left(\mathrm{Cl}_{K X} F \backslash F\right) \backslash T$. Then $T$ is closed in $K X$. Since $K X \backslash X$ is relatively 0 -dimensionally embedded in $K X$, there is an sb open set $U$ of $K X$ such that $p \in U$ and $\left(\mathrm{Cl}_{K X} U\right) \cap T=\varnothing$. Consider the open set $U \cap \mathrm{Cl}_{K X} F$ of $\mathrm{Cl}_{K X} F$. The boundary in $\mathrm{Cl}_{K X} F$ of $U \cap \mathrm{Cl}_{K X} F$ is

$$
\begin{aligned}
& \mathrm{Cl}_{K X}\left(U \cap \mathrm{Cl}_{K X} F\right) \backslash U \cap \mathrm{Cl}_{K X} F \subset\left[\mathrm{Cl}_{K X}\left(U \cap \mathrm{Cl}_{K X} F\right) \backslash U\right] \cap \mathrm{Cl}_{K X} F \\
& \quad \subset\left[\left(\mathrm{Cl}_{K X} U\right) \backslash U\right] \cap \mathrm{Cl}_{K X} F \subset \mathrm{bd}_{K X} U \cap \mathrm{Cl}_{K X} F \\
& \quad \subset X \cap \mathrm{Cl}_{K X} F=F .
\end{aligned}
$$

Then $U \cap \mathrm{Cl}_{K X} F$ is an sb open subset of $\mathrm{Cl}_{K X} F$ and a neighbourhood (in $\left.\mathrm{Cl}_{K X} F\right)$ of $p$, while $T \cap\left(\mathrm{Cl}_{K X} F\right) \cap U=\varnothing$. Thus each point of $\mathrm{Cl}_{K X} F \backslash F$ has a basis of sb open sets of $\mathrm{Cl}_{K X} F$. Hence $\mathrm{Cl}_{K X} F \backslash F$ is relatively 0-dimensionally embedded in $\mathrm{Cl}_{K X} F$. It follows from 1.8 that $F$ is almost rimcompact.

3.5. EXAMPLE. Choose $\mathscr{R}$ to be a maximal almost disjoint collection of infinite subsets of $\mathscr{N}$ such that $\beta(\mathscr{N} \cup \mathscr{R}) \backslash(\mathscr{N} \cup \mathscr{R})$ is homeomorphic to $I$. Let $Z=\left[\beta(\mathscr{N} \cup \mathscr{R}) \times\left(\omega_{1}+1\right)\right] \backslash\left[(\mathscr{N} \cup \mathscr{R}) \times\left\{\omega_{1}\right\}\right]$, and $X=$ $Z \backslash\left\{\left(\frac{1}{2}, \omega_{1}\right)\right\}$. Then $X$ is an open subset of $Z$. As demonstrated in 3.8 of [2], $X$ is not a 0 -space, while according to $1.9, Z$ is almost rimcompact.

For completeness we include the following example which illustrates that the product of two rimcompact spaces need not be a 0 -space. We mention that it is straightforward to show that a space possessing a compactification with countable remainder is rimcompact. 
3.6. ExAMPLE. Choose $\mathscr{R}$ to be a family such that $\beta(\mathscr{N} \cup \mathscr{R}) \backslash \mathscr{N} \cup \mathscr{R}$ $=I$. Let $P, Q$ denote the irrationals and rationals in $I$, respectively. If $Y=\mathscr{N} \cup \mathscr{R} \cup P$, then $\beta Y \backslash Y=Q$, hence $Y$ is rimcompact. According to $1.3 \beta((\mathscr{N} \cup \mathscr{R}) \times(\mathscr{N} \cup \mathscr{R}))=\beta(\mathscr{N} \cup \mathscr{R}) \times \beta(\mathscr{N} \cup \mathscr{R})$, so by 1.4 $\beta(Y \times Y)=\beta Y \times \beta Y$. Let $Z=\beta(Y \times Y) \backslash(Y \times Y)$. If $q \in Q$, let $C_{(q, q)}$ denote the connected component of $(q, q)$ in $Z$. We show that $C_{(q, q)}$ is not compact, hence $Y \times Y$ is not a 0 -space. Now $q \times I$ is a connected subset of $Z$. For each $q^{\prime} \in Q, I \times q^{\prime}$ is a connected subset of $Z$ which intersects $q \times I$, hence $\bigcup_{q^{\prime} \in Q}\left(I \times q^{\prime}\right) \subseteq C_{(q, q)}$. The smallest compact connected set containing $\cup_{q^{\prime} \in Q}\left(I \times q^{\prime}\right)$ is $I \times I$. However, $(I \times I) \cap(Y \times Y) \neq \varnothing$, hence $C_{(q, q)}$ is not compact.

4. Images and preimages. Continuous images and preimages of rimcompact spaces need not be rimcompact, even if the map is perfect. In fact, since any completely regular space is the image of an extremally disconnected space (i.e., a space in which disjoint open sets have disjoint closures) under a perfect irreducible map (see [11]), the perfect image of a rimcompact space need not even be a 0 -space. The next example shows that the perfect preimage of a rimcompact space need not be a 0 -space. However, we show in 4.3 that if the perfect preimage of an almost rimcompact space is a 0 -space, then that preimage is almost rimcompact.

4.1. EXAmple. Let $Y=I \times\{0,1,1 / 2,1 / 3, \ldots\}$, and

$$
X=\left[Y \times\left(\omega_{1}+1\right)\right] \backslash\left[I \times\left\{1, \frac{1}{2}, \frac{1}{3}, \ldots\right\} \times\left\{\omega_{1}\right\}\right] .
$$

It is shown in 3.7 of [2] that $X$ is not a 0 -space. Let

$$
f: I \times\left\{0,1, \frac{1}{2}, \frac{1}{3}, \ldots\right\} \times\left(\omega_{1}+1\right) \rightarrow\left\{0,1, \frac{1}{2}, \frac{1}{3}, \ldots\right\} \times\left(\omega_{1}+1\right)
$$

be the projection map. Then $f$ is closed, since $I$ is compact. Let

$$
S=\left[\left\{0,1, \frac{1}{2}, \frac{1}{3}, \ldots\right\} \times\left(\omega_{1}+1\right)\right] \backslash\left[\left\{1, \frac{1}{2}, \frac{1}{3}, \ldots\right\} \times\left\{\omega_{1}\right\}\right] .
$$

Since $f^{\leftarrow}(y)=I \times\{y\}$, for $y \in S, f$ is a perfect map from $X$ onto $S$. The space $S$, being a subspace of $\left\{0,1, \frac{1}{2}, \frac{1}{3}, \ldots\right\} \times\left(\omega_{1}+1\right)$, is 0 -dimensional (and hence rimcompact).

The following is 1.2 of [6].

4.2. LemMA. Let $f: X \rightarrow Y$ be a perfect map. If $S$ is a compact subset of $Y$, then $f^{\leftarrow}[S]$ is a compact subset of $X$.

4.3. TheOREM. Let $f: X \rightarrow Y$ be a perfect map. If $X$ is a 0 -space, and $Y$ is almost rimcompact, then $X$ is almost rimcompact. 
Proof. We show that $X$ is quasi-rimcompact. It then follows from 1.8 that $X$ is almost rimcompact. If $x \in R(X)$, let $K_{x}=f^{\leftarrow}[K]$, where $K$ is the compact subset of $Y$ witnessing the fact that $Y$ is quasi-rimcompact at $f(x)$. According to 4.2, $K_{x}$ is a compact subset of $X$. Suppose that $F$ is a closed subset of $X$ such that $F \cap K_{x}=\varnothing$. Then $K \cap f[F]=\varnothing$. Since $f$ is a closed map, it follows from our choice of $K$ that there is a $\pi$-open subset $W$ of $Y$ such that $f(x) \in W \subseteq \mathrm{Cl}_{Y} W \subset Y \backslash f[F]$. As $f$ is a perfect map, and $\mathrm{bd}_{Y} W$ is compact, according to $4.2 f^{\leftarrow}\left[\mathrm{bd}_{Y} W\right]$ is compact. Since bd $_{X} f^{\leftarrow}[W] \subset f^{\leftarrow}\left[\mathrm{bd}_{X} W\right], f^{\leftarrow}[W]$ is a $\pi$-open subset of $X$. Also, $x \in f^{\leftarrow}$ $[W]$, and $F \cap \mathrm{Cl}_{X} f^{\leftarrow}[W]=\varnothing$. Thus $x$ and $F$ are $\pi$-separated. Hence $X$ is quasi-rimcompact, and the theorem is proved.

In 4.3, $X$ and $Y$ can be chosen so that $X$ is not rimcompact and $Y$ is rimcompact.

4.4. EXAmple. Choose $\mathscr{R}$ to be a family such that $\beta(\mathscr{N} \cup \mathscr{R}) \backslash \mathscr{N} \cup \mathscr{R}$ is homeomorphic to $I$. Then $F(\mathscr{N} \cup \mathscr{R})=\omega(\mathscr{N} \cup \mathscr{R})$, the one-point compactification of $\mathscr{N} \cup \mathscr{R}$. If

$$
X=\left[\beta(\mathscr{N} \cup \mathscr{R}) \times\left(\omega_{1}+1\right)\right] \backslash\left[(\mathscr{N} \cup \mathscr{R}) \times\left\{\omega_{1}\right\}\right],
$$

then according to $1.9, X$ is almost rimcompact but is not rimcompact. Let

$$
f: \beta(\mathscr{N} \cup \mathscr{R}) \times\left(\omega_{1}+1\right) \rightarrow \omega(\mathscr{N} \cup \mathscr{R}) \times\left(\omega_{1}+1\right)
$$

be the natural map, and let

$$
Z=\left[\omega(\mathscr{N} \cup \mathscr{R}) \times\left(\omega_{1}+1\right)\right] \backslash\left[(\mathscr{N} \cup \mathscr{R}) \times\left\{\omega_{1}\right\}\right] .
$$

If $z \in Z$, then $f^{\leftarrow}(z)=\{z\}$ or $f^{\leftarrow}(z)=I \times\{p\}$ for some $p \in\left(\omega_{1}+1\right)$. Also $f^{\leftarrow}[Z]=X$, so $\left.f\right|_{X}$ is a perfect map from $X$ into $Z$. The space $Z$ is 0 -dimensional (and hence rimcompact).

It is well known that if $f: X \rightarrow Y$ is a map, where $X$ and $Y$ are 0 -dimensional, then $f$ extends to $g \in C(F X, F Y)=C\left(\beta_{0} X, \beta_{0} Y\right)$. The following generalizes this fact.

4.5. Theorem. Suppose that $X$ is a space, $Y$ is 0 -dimensional and $K X$ is a perfect compactification of $X$. If $f: X \rightarrow Y$ is a map, then $f$ extends to $g \in C\left(K X, \beta_{0} Y\right)$.

Proof. Subsets $C$ and $D$ of $Y$ have disjoint closures in $\beta_{0} Y$ if and only if $C$ and $D$ are contained in disjoint clopen subsets $U$ and $Y \backslash U$ of $Y$ respectively. Since $f^{\leftarrow}[U], f^{\leftarrow}[Y \backslash U]$ are then disjoint clopen subsets of 
$X$, and $K X$ is a perfect compactification of $X$, it follows that $\mathrm{Cl}_{K X} f^{\leftarrow}[U]$ $\cap \mathrm{Cl}_{K X} f^{\leftarrow}[Y \backslash U]=\varnothing$. Then $\mathrm{Cl}_{K X} f^{\leftarrow}[C] \cap \mathrm{Cl}_{K X} f^{\leftarrow}[D]=\varnothing$; thus by $1.1, f$ extends to $g \in C\left(K X, \beta_{0} Y\right)$.

4.6. Definition. A map $f: X \rightarrow Y$ is monotone if $f^{\leftarrow}(y)$ is connected for each $y \in Y$.

The following answers a question communicated verbally to R. G. Woods (Topology Conference, 1980) by D. Bellamy.

4.7. TheOREM. Let $f: X \rightarrow Y$ be a monotone quotient map, and let $K X$, $K Y$ be perfect compactifications of $X$ and $Y$ respectively. If $f$ extends to $g \in C(K X, K Y)$, then $g$ is monotone.

Proof. Suppose that there is $p \in K Y$ such that $g^{\leftarrow}(p)$ is not connected. Write $g^{\leftarrow}(p)=G_{1} \cup G_{2}$, where $G_{1}$ and $G_{2}$ are disjoint closed subsets of $g^{\leftarrow}(p)$. Since $g^{\leftarrow}(p)$ is compact, $G_{1}$ and $G_{2}$ are disjoint compact subsets of $K X$; hence there are open sets $U_{1}$ and $U_{2}$ of $X$ such that $G_{i} \subset E x_{K X} U_{i}(i=1,2)$ and $\mathrm{Cl}_{K X} U_{1} \cap \mathrm{Cl}_{K X} U_{2}=\varnothing$. Since $g$ is a closed map, there is an open set $V$ of $Y$ such that $g \leftarrow(p) \subset g \leftarrow[V] \subset$ $E x_{K X} U_{1} \cup E x_{K X} U_{2}$. Let $W_{l}=g^{\leftarrow}[V] \cap U_{i}=f^{\leftarrow}[V \cap Y] \cap U_{l}(i=1,2)$. Then $W_{1}$ and $W_{2}$ are disjoint open subsets of $X$, and $W_{1} \cup W_{2}=f^{\leftarrow}[V \cap$ $Y]$. Since $f^{\leftarrow}(y)$ is connected for each $y \in Y, W_{l}=f^{\leftarrow}\left[V_{l}\right]$ for some subset $V_{l}$ of $Y(i=1,2)$. Since $f$ is a quotient map, $V_{i}$ is open in $Y$ $(i=1,2)$. Then $V \cap Y=V_{1} \cup V_{2}$, while $V_{1} \cap V_{2}=\varnothing$. It follows from 1.5 (i) and (ii), and 1.6 that $p \in E x_{K Y} V=E x_{K Y} V_{1} \cup E x_{K Y} V_{2}$, while $E x_{K Y} V_{1} \cap E x_{K Y} V_{2}=\varnothing$. Suppose without loss of generality that $p \in$ $E x_{K Y} V_{1}$. Since $g \leftarrow\left[E x_{K Y} V_{1}\right]$ is an open subset of $K X$ containing $f^{\leftarrow}\left[V_{1}\right]$,

$$
g^{\leftarrow}(p) \subset g^{\leftarrow}\left[E x_{K Y} V_{1}\right] \subset E x_{K X} f^{\leftarrow}\left[V_{1}\right]=\operatorname{Ex}_{K X} W_{1} \subset E x_{K X} U_{1},
$$

which contradicts the fact that $g^{\leftarrow}(p) \cap \operatorname{Ex}_{K X} U_{2} \neq \varnothing$. Thus $g^{\leftarrow}(p)$ is connected for each $p \in K Y$.

4.8. Corollary. Suppose that $X$ is a 0 -space and $Y$ is 0 -dimensional. If there is a perfect monotone map from $X$ into $Y$, then $X$ is almost rimcompact and $F_{0} X \backslash X$ is homeomorphic to $F Y \backslash Y$.

Proof. Let $f: X \rightarrow Y$ be a perfect monotone map. Then $f$ extends to $g \in C\left(F_{0} X, F Y\right)$ by 4.5 . Since $f$ is perfect, $g{ }^{\leftarrow}[F Y \backslash Y]=F_{0} X \backslash X$. As $f$ is monotone, it follows from 4.7 that $g \leftarrow(y)$ is connected for each $y \in F Y \backslash Y$. Since $F_{0} X \backslash X$ is 0 -dimensional, and $g^{\leftarrow}(y) \subset F_{0} X \backslash X$, 
$\left|g^{\leftarrow}(y)\right|=1$. Thus $\left.g\right|_{F_{0} X \backslash X}: F_{0} X \backslash X \rightarrow F Y \backslash Y$ is a closed continuous one-to-one map, hence $g$ is a homeomorphism. The fact that $X$ is almost rimcompact follows from 4.3.

Example 4.1 shows that the perfect monotone preimage $X$ of a 0 -dimensional space need not be a 0 -space, while Example 4.4 shows that even if $X$ is a 0 -space, $X$ need not be rimcompact.

\section{REFERENCES}

[1] R. E. Chandler, Hausdorff compactifications, Lecture Notes in Pure and Applied Mathematics No. 23, Marcel Dekker, Inc., New York and Basel, 1976.

[2] B. Diamond, Almost rimcompact spaces, submitted.

[3] R. Engelking, General Topology, Polish Scientific Publishers, Warsaw, 1977.

[4] L. Gillman and M. Jerison, Rings of Continuous Functions, Van Nostrand, New York, 1960.

[5] I. Glicksberg, Stone-Cech compactification of products, Trans. Amer. Math. Soc., 90 (1959), 369-382.

[6] M. Henriksen and J. R. Isbell, Some properties of compactifications, Duke Math. J., 25 (1958), 83-105.

[7] J. R. Isbell, Uniform spaces, Amer. Math. Soc. Math. Surveys No. 12 (1962).

[8] J. R. McCartney, Maximum zero-dimensional compactifications, Proc. Camb. Phil. Soc., 68 (1970), 653-661.

[9] K. Morita, On bicompactifications of semibicompact spaces, Sci. Rep. Tokyo Bunrika Daigaku Sec. A vol. 4, 94 (1952).

[10] E. G. Sklyarenko, Some questions in the theory of bicompactifications, Amer. Math. Soc. Trans., 58 (1966), 216-244.

[11] D. P. Strauss, Extremally disconnected spaces, Proc. Amer. Math. Soc., 18 (1967), 305-309.

[12] J. Terasawa, Spaces $\mathscr{N} \cup \mathscr{R}$ and their dimensions, Topology Appl., 11 (1980), 93-102.

Received October 3, 1983 and in revised form January 9, 1984. This research was partially supported by a scholarship from the Natural Sciences and Engineering Research Council of Canada, and constitutes a portion of the author's doctoral dissertation.

UNIVERSITY OF MANITOBA

WINNIPEG, MANITOBA R3T 2N2

CANADA 



\title{
PACIFIC JOURNAL OF MATHEMATICS EDITORS
}

DONALD BABBITT (Managing Editor)
University of California
Los Angeles, CA 90024
CHARLEs R. DEPrIMA
California Institute of Technology
Pasadena, CA 91125
R. FINN
Stanford University
Stanford, CA 94305

DoNAld BABBitT (Managing Editor)

Hermann FlaschKa

University of Arizona

Tucson, AZ 85721

RAmesh A. Gangolli

University of Washington

Seattle, WA 98195

ROBION KIRBY

University of California

Berkeley, CA 94720

C. C. MOORE

University of California

Berkeley, CA 94720

\author{
Hugo Rossi \\ University of Utah \\ Salt Lake City, UT 84112 \\ H. SAMELSON \\ Stanford University \\ Stanford, CA 94305 \\ HAROLD STARK \\ University of California, San Diego \\ La Jolla, CA 92093
}

ASSOCIATE EDITORS

R. ARENS

E. F. BECKENBACH
(1906-1982)

B. H. NEUMANN

F. WOLF

K. Yoshida

\section{SUPPORTING INSTITUTIONS}

UNIVERSITY OF ARIZONA

UNIVERSITY OF BRITISH COLUMBIA

CALIFORNIA INSTITUTE OF TECHNOLOGY

UNIVERSITY OF CALIFORNIA

MONTANA STATE UNIVERSITY

UNIVERSITY OF NEVADA, RENO

NEW MEXICO STATE UNIVERSITY

OREGON STATE UNIVERSITY
UNIVERSITY OF OREGON

UNIVERSITY OF SOUTHERN CALIFORNIA

STANFORD UNIVERSITY

UNIVERSITY OF HAWAII

UNIVERSITY OF TOKYO

UNIVERSITY OF UTAH

WASHINGTON STATE UNIVERSITY

UNIVERSITY OF WASHINGTON 


\section{Pacific Journal of Mathematics}

\section{Vol. 118, No. $1 \quad$ March, 1985}

Dan Amir, On Jung's constant and related constants in normed linear spaces ...1 Abdul Aziz, On the location of the zeros of certain composite polynomials . . 17 Joseph Barback, On hereditarily odd-even isols and a comparability of

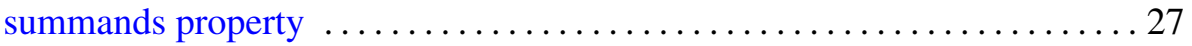

Matthew G. Brin, Klaus Johannson and Peter Scott, Totally peripheral 3-manifolds ........................................ 37

Robert F. Brown, A topological bound on the number of distinct zeros of an

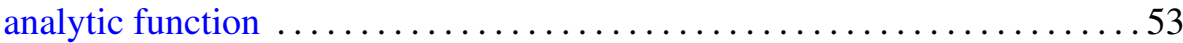

K. C. Chattopadhyay, Not every Lodato proximity is covered .......... 59

Beverly Diamond, Some properties of almost rimcompact spaces . .......63 63

Manfred Dugas and Rüdiger Göbel, On radicals and products . ......... 79

Abdelouahab El Kohen, A hyperbolic problem .................. 105

Harry Gonshor, Remarks on the Dedekind completion of a nonstandard model of the reals ................................... 117

William H. Kazez, On equivalences of branched coverings and their action

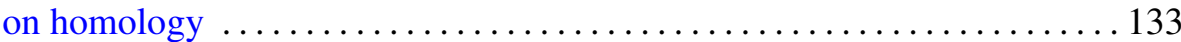

Darrell Conley Kent, On the Wallman order compactification .......... 159

Martin Andrew Magid, Lorentzian isoparametric hypersurfaces . . . . . . 165

Milan Miklavčič, Stability for semilinear parabolic equations with noninvertible linear operator

Richard Dean Neidinger and Haskell Paul Rosenthal, Norm-attainment of linear functionals on subspaces and characterizations of Tauberian operators

Johannes Vermeer, Closed subspaces of $H$-closed spaces 